\title{
Novel Approaches to Treatment of Autoimmune Neuroinflammation and Lessons for Drug Development
}

\author{
Eran Nizri $^{\mathrm{a}} \quad$ Michal Irony-Tur-Sinai ${ }^{\mathrm{a}} \quad$ Nikolaos Grigoriadis $^{\mathrm{c}}$ Oded Abramsky ${ }^{\mathrm{a}}$ \\ Gabi Amitai $^{\text {b }}$ Talma Brenner ${ }^{\text {a }}$ \\ a Laboratory of Neuroimmunology, Department of Neurology, Agnes-Ginges Center for Human Neurogenetics, \\ Hadassah-Hebrew University Medical Center, Jerusalem; ${ }^{b}$ Division of Medicinal Chemistry, IIBR, Ness Ziona, Israel; \\ 'Department of Neurology, Aristotle University, Thessaloniki, Greece
}

\section{Key Words}

Central nervous system inflammation • Multiple sclerosis •

Experimental autoimmune encephalomyelitis · Drug

development $\cdot$ Bifunctional molecules $\cdot$ Natural

immunomodulating protein $\cdot \alpha$-Fetoprotein

\begin{abstract}
Drug development, and especially that intended for central nervous system (CNS) disorders, still poses a challenge. We investigated both the use of bifunctional compounds designed for multiple targeting and enhanced CNS permeability, and of recombinant $\alpha$-fetoprotein (AFP), a natural pregnancy-associated immunomodulating protein for the treatment of CNS inflammation. Bifunctional compounds showed a novel pharmacokinetic profile due to the conjugation, yet retained, and even improved pharmacodynamics. AFP was well tolerated and decreased various aspects of neuroinflammation, including disease severity, axonal loss and damage, T-cell reactivity, and antigen presentation. Our results show that both strategies may serve as future drug modalities.

Copyright $\odot 2007$ S. Karger AG, Basel
\end{abstract}

\section{Introduction}

The relatively small number of new drugs developed in recent years is a cause for concern. The number of new molecular entities, drugs targeted to novel molecular sites and thus with a possible disease-modifying property, has decreased from 52 in 1996 to 12 in 2005 [1]. This is in contrast to the doubling in $\mathrm{R} \& \mathrm{D}$ expenses during the same period [2]. Hopes that the sequencing of the human genome would expedite the process of drug discovery were disproved. Although the combination of the human genome project with powerful computational techniques can theoretically identify new genes encoding for possible drug targets, and is now being applied to the drug discovery process [3], the desired increase in approvals has not been met. Overall, the reduced number of new molecular entities has a great impact on the price of new drugs $[1,2,4]$. Treatment of central nervous system (CNS) inflammation poses an even more difficult challenge, due to the need for the active molecule to penetrate the blood-brain barrier and act within the CNS.

\section{KARGER}

Fax +4161306 1234 E-Mail karger@karger.ch www.karger.com
(C) 2007 S. Karger AG, Basel

0031-7012/07/0791-0042\$23.50/0

Accessible online at:

www.karger.com/pha
Talma Brenner

Department of Neurology, Hadassah Hebrew University Medical Center

PO Box 12000, Jerusalem 91120 (Israel)

Tel. +972 2677 6957, Fax +972 26437782

E-Mail brenner@cc.huji.ac.il 


\section{Multiple Sclerosis and Experimental Autoimmune Encephalomyelitis}

Our approach to the development of novel treatment for autoimmune neuroinflammation is based on experiments in the animal model of multiple sclerosis (MS), i.e., experimental autoimmune encephalomyelitis (EAE). EAE is an inflammatory demyelinating autoimmune disease of the CNS. Since the disease is induced by a known antigen, study of the pathogenesis of EAE has led to many immunological insights, allowing lessons from EAE to be generalized and applied to other autoimmune diseases [5]. Experiments in EAE led to the development of several approved drugs for MS and the model has been useful in helping us to understand the disease. It was found that the inflammatory process in EAE and MS involves extravasation of activated $\mathrm{T}$ cells through blood vessels and activation of CNS immunocompetent cells, which accumulate at the inflammatory sites. The initial inflammatory phase is followed by a phase of selective demyelination and, finally, neurodegeneration $[6,7]$. The latter is mainly characterized by axonal injury, which begins at disease onset and correlates with the degree of inflammation within lesions. A threshold of axonal loss must be exceeded before irreversible neurological disability occurs [8]. In spite of the marked progress in the assessment and therapy of MS, there is a need for improved therapy that will halt disease progression and lead to reversal of the neurological disability.

\section{Treatment of Neuroinflammation with Bifunctional Compounds Eliciting Both Anti-Inflammatory and Cholinergic Activity}

The design of these molecules is based on a combination of two different pharmacologically active entities in one molecule. One moiety is a non-steriodal anti-inflammatory drug (NSAID, e.g. ibuprofen, diclofenac, naproxen or indomethacin), the other is either an acetylcholinesterase (AChE) inhibitor or a cholinergic agonist. These two moieties are coupled by a hydrocarbon chain spacer which contains 8-10 carbon atoms that enhances the lipophilicity of the active moieties and provides better penetration through the blood-brain barrier. Some of these bifunctional compounds (e.g. ibuprofen-octyl-pyridostigmine, IBU-PO) reduced lesion volume and limited neuronal injury in closed head injury and increased survival in hypobaric hypoxia [9]. In another study, IBU-PO inhibited, at submicromolar concentrations, the toxicity exerted by $\beta$-amyloid peptide on neuronal rat brain cells by acting as a glycogen-synthase-kinase-3 $\beta$ (GSK-3 $\beta$ ) inhibitor [10]. As the bifunctional molecules showed promising results in these settings, in which there is a prominent inflammatory component, we tested these compounds in EAE. Moreover, as EAE is a complex inflammatory process involving various active cells and immunological damage mechanisms, we thought it would be useful to target different cascades in its pathogenesis. Recent data question the strategy of drug selectivity, i.e. drugs designed to interact with a single molecular entity, and call for molecules that will address several molecular targets [11]. Single targets proved inefficient in many cases because of biological redundancy: meaningful physiological and pathological processes are not dependent solely on one molecular target. Indeed, it was shown that combination therapy in EAE was more effective than either of the treatments alone, and that multiple treatment regimens cannot only prevent damage in animals but may even reverse it [12]. This was our primary incentive in the use of bifunctional molecules. We tested two bifunctional compounds, IBU-PO and IBU-PD, composed of a NSAID moiety, ibuprofen (IBU), and a cholinergic moiety, the AChE inhibitor pyridostigmine (pyridostigmine octyl- PO, pyridostigmine decyl$\mathrm{PD}$, respectively) [13]. The two compounds differ in the length of the hydrophobic hydrocarbon spacer connecting the two moieties. IBU-PD, in which the spacer spans ten carbon atoms, is more hydrophobic than IBU-PO, which contains a hydrocarbon spacer consisting of eight carbon atoms (fig. 1a, b). Consequently, IBU-PD is better able to penetrate the CNS. IBU can ameliorate EAE through cyclooxygenase (COX)-dependent mechanisms, i.e. inhibition of arachidonic acid metabolite formation [14]. IBU was previously shown to reduce the production of insoluble $\beta$-amyloid peptide although not by COX inhibition [15]. IBU also reduced NF- $\kappa \mathrm{B}$ transcription activity by a non-COX-dependent mechanism [16]. This reduction can influence the expression of iNOS and COX-2 [17], thus lowering $\mathrm{NO}$ and $\mathrm{PGE}_{2}$ production, respectively. In addition, it was shown that other NSAIDs reduced TNF- $\alpha$ production in the CNS [18], a finding particularly relevant to our bifunctional compound, which was designed to act in the CNS. The cholinergic moiety in the bifunctional compounds leads to the activation of the $\alpha 7$ nicotinic acetylcholine receptor (nAChR) in immune cells. Anti-inflammatory properties were attributed to this receptor in macrophages [19] and lymphocytes [20]. We have shown that AChE inhibitor may activate this anti-inflammatory receptor by slowing ACh breakdown 


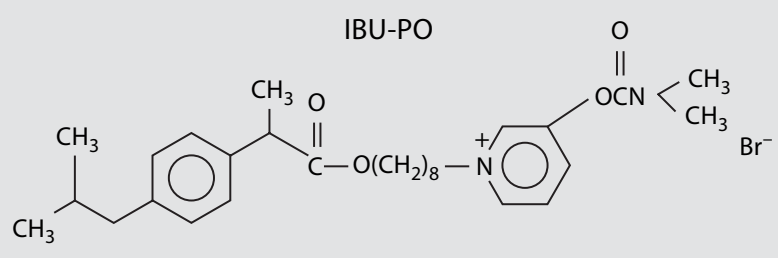

a

IBU-PD

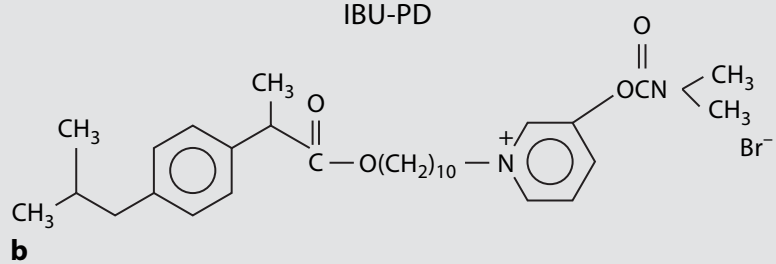

IBU-octyl-cytisine

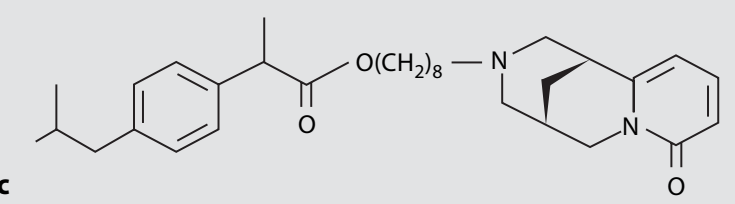

Fig. 1. The chemical structure of bifunctional compounds tested in our studies.

and thus increasing the number of potential endogenous ligands [20]. Therefore, the use of the bifunctional molecules would allow the targeting of either the COX-dependent or COX-independent inflammatory cascade and of the cholinergic immune system, along with increased permeability into the CNS. We previously found that the molecular combination of the two functions in one molecule does not affect the inhibition of $\mathrm{AChE}$ or the inhibition of COX isoenzymes [9].

\section{Effects of the Bifunctional Molecules on Clinical}

Parameters of EAE, T-Cell Reactivity and

the Inflammatory Response of CNS Resident

Immunocompetent Cells

Treatment with both IBU-PO (1 mg/kg, i.p.) and IBUPD $(0.1 \mathrm{mg} / \mathrm{kg}$, i.p.) from the day of EAE induction ameliorated the clinical outcome, as manifested here by a significant (30-40\%) reduction in disease severity (fig. 2a). Treatment with IBU $(0.1 \mathrm{mg} / \mathrm{kg})$ or PD $(0.1 \mathrm{mg} / \mathrm{kg})$ alone did not reduce the disease burden: the mean severity in the placebo control group was 1.9, and in the IBU- and PD-treated groups 1.9 and 2.1, respectively. The ineffec-

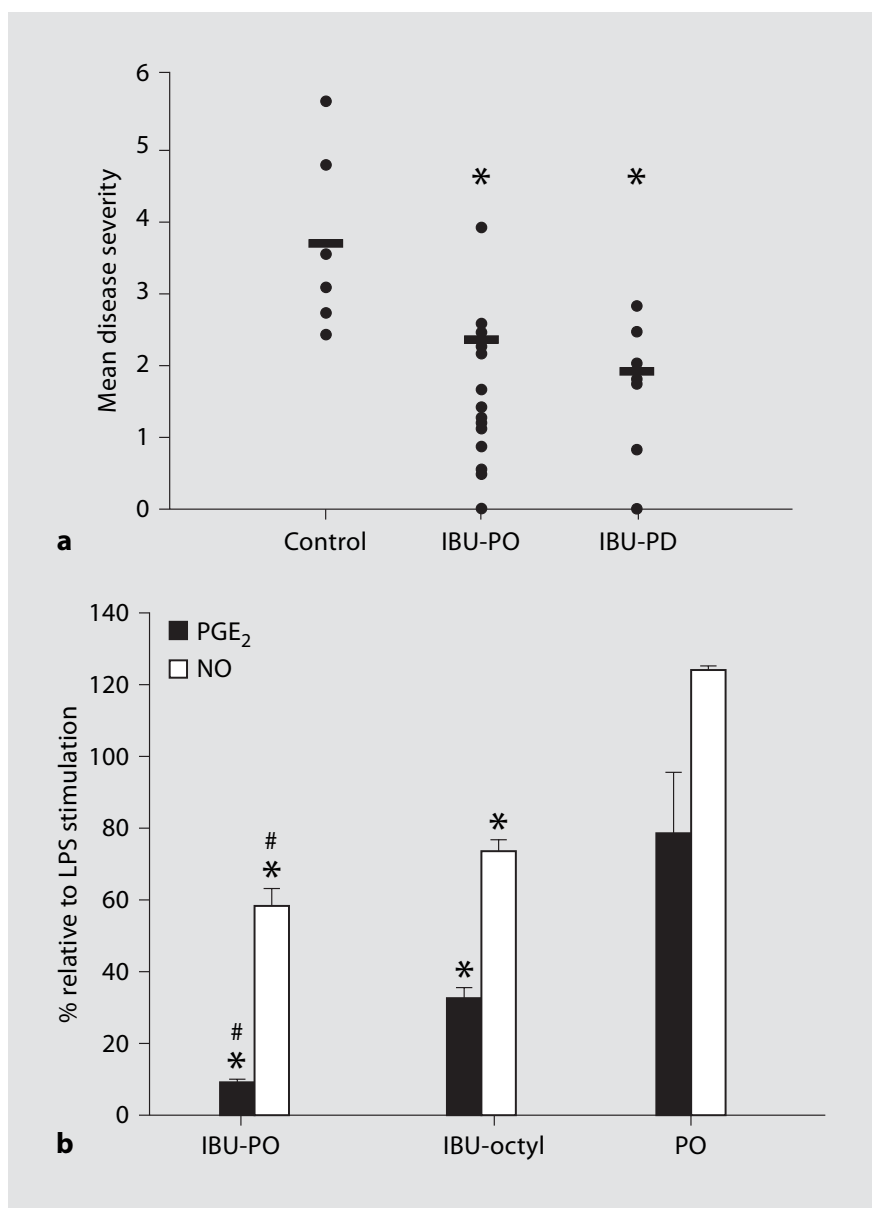

Fig. 2. Effects of bifunctional molecules on models of CNS inflammation. a IBU-PO and IBU-PD ameliorated clinical symptoms of EAE. Daily treatment from the day of disease induction with IBU-PO ( $1 \mathrm{mg} / \mathrm{kg}, \mathrm{n}=9)$ and IBU-PD $(0.1 \mathrm{mg} / \mathrm{kg}, \mathrm{n}=8)$ significantly ameliorated disease severity in comparison with that in control animals (administered the same volume of vehicle, $\mathrm{n}=8$ ). ${ }^{*} \mathrm{p}<0.05$. The results are expressed as mean clinical score for each mouse. $\mathbf{b}$ Inhibition of inflammatory mediators production in primary culture of activated astrocytes by IBU-PO. The results are presented as the percent of $\mathrm{NO}$ and $\mathrm{PEG}_{2}$ production in LPSstimulated cells. ${ }^{*} \mathrm{p}<0.05$ vs. LPS, ${ }^{*} \mathrm{p}<0.05$ vs. IBU-octyl-treated cells.

tiveness of IBU under these conditions is attributable to its low dose and limited availability in the CNS (see below). The same pattern of synergistic effect achieved by the bifunctional compound and not by either of its components, was also observed in T-cell reactivity towards the encephalitogenic peptide myelin oligodendrocyte glycoprotein (MOG) that was used for disease induction [13].

The effects of IBU-PO on activated astrocytes in primary culture were also studied, as these cells become ac- 
tivated in the inflamed CNS in general, and in EAE in particular. When stimulated by an inflammatory factor such as LPS or TNF- $\alpha$, astrocytes produce various cytokines and inflammatory mediators, including $\mathrm{PGE}_{2}$. In this system, IBU-PO had a greater impact than IBU on $\mathrm{PGE}_{2}$ production, although $\mathrm{PO}$ and also other AChE inhibitors tested had no effect (fig. 2b). This finding indicated a novel and unexpected influence of the bifunctional compound. The addition of a second moiety augmented the effect of the active IBU moiety. This effect is not attributable merely to the increased permeability of IBU-PO, since IBU-octyl contains the hydrocarbon spacer responsible for enhanced lipophilicity, but is less active in $\mathrm{PGE}_{2}$ suppression. Furthermore, we assume that cells are more permeable to IBU-octyl than to IBU-PO, since it does not contain the positively charged quaternary nitrogen present in pyridostigmine. The use of IBU-PO may possibly affect the cellular metabolism of the active substance, as no change was seen in COX inhibition by the bifunctional compound compared with that of IBUoctyl alone.

We tested another bifunctional molecule, IBU-octylcytisine (fig. 1c), in which the cholinergic moiety is cytisine, a nicotinic agonist. This molecule was designed to interact directly with $\alpha 7 \mathrm{AChR}$ instead of inhibiting ACh breakdown. Our results show that daily treatment with IBU-octyl-cytisine $(2.5 \mathrm{mg} / \mathrm{kg}$, i.p.) markedly inhibited (by 70\%) clinical and pathological parameters of EAE. The clinical score of animals treated with either of the moieties (i.e. IBU-octyl or cytisine, in equivalent doses) and of animals treated with both moieties unconjugated but mixed together in solution, was virtually the same as that of the control. Treatment with cytisine was severely toxic, with $80 \%$ mortality, yet none of the animals treated with the bifunctional compound showed any significant signs of toxicity.

The strategy in using bifunctional compounds rests on several assumptions: (1) The activity of each of the moieties is preserved in the bifunctional compounds. (2) The utilization of multiple targets in the treatment of the pathologic process (EAE, in our case) is more efficient than treatment aimed at one target. (3) The effect of the bifunctional compound is superior to that of a non-conjugated mixture of the two active moieties, i.e., the use of bifunctional compounds is superior to conventional combination therapy. The data indeed show that careful selection of the appropriate active moieties allows preservation of the two activities. Treatment with either of the moieties or in a mixture was significantly less effective than treatment with the bifunctional molecules. Further- more, the use of the bifunctional compounds changed the pharmacokinetics of the active moieties while preserving their pharmacodynamics. In addition, the unitary pharmacokinetics of the bifunctional compound mobilizes both moieties to the target at the same time. The activity of the IBU moiety in IBU-PO was augmented, whereas the toxicity of cytisine was diminished by IBU-octyl-cytisine. The use of the bifunctional compounds allowed a significant reduction in IBU dose. Previous reports on the beneficial effect of COX inhibitors on EAE dealt with a two orders of magnitude higher dose (200 mg/kg) [14] than in our study $(0.1-1 \mathrm{mg} / \mathrm{kg})$. A reduced dose of IBU is advantageous in chronic treatment in light of its adverse gastrointestinal and other side effects.

The significance of compliance and adherence to treatment in the clinical setting cannot be overemphasized [21-23]. Compliance may affect the treatment protocol and lead to disappointing clinical outcomes even if the drug is of proven biological efficacy. The use of bifunctional compounds can improve compliance in patients: not only can several drugs be taken in one pill, but the daily dosage can be decreased due to the altered metabolism of the new compound.

The moieties incorporated in our bifunctional compounds are known off-patent drugs. The use of such compounds allows accessibility to a large database regarding their pharmacologic profile. Since they have been approved and are in wide clinical use, specific receptor interactions, drug metabolism and excretion, adverse effects and dosage have been determined. Thus we suggest amplifying the efficacy of known drugs by the bifunctional combination strategy - a new way 'to teach old drugs new tricks' [24].

\section{Treatment of Autoimmune Neuroinflammation by the Natural Immunomodulating Protein $\alpha$-Fetoprotein}

The strategy of endogenous/natural compounds as therapeutic agents has several advantages. Harnessing physiological mechanisms to achieve medical outcomes enjoys the efficacy of normal functioning. Once a hormonal cascade has been elucidated, each step can be deliberately used to achieve effectively a given purpose. Moreover, by mimicking the physiological environment, the toxicity of treatment is circumvented, as manifested by reduced side effects. The immunogenicity obstacle is also bypassed. A naturally active protein is readily available for the pharmaceutical industry, based on cloning 
and translational techniques. Clinical examples of this strategy are: treatment with follicular-stimulating hormone and luteinizing hormone for induction of superovulation in assisted reproduction [25], growth hormone supplementation for developmental deficits [26], etc. In addition, much research has been devoted to the immunomodulating activity of estrogen, a steroidal hormone present at high levels during pregnancy [27]. We here present a new application of this strategy, namely the use of $\alpha$-fetoprotein (AFP) to mimic the pregnancy-associated immune modulation.

Clinical remissions during the second half of pregnancy, as well as postpartum relapses, have been described in several autoimmune diseases, such as myasthenia gravis (MG), rheumatoid arthritis, thyroiditis and MS [28-32]. The suppressive effect of pregnancy on autoimmune conditions may be correlated with other immunological phenomena occurring during pregnancy, such as the immunosuppressive effect of both maternal serum and amniotic fluid on lymphocyte and antibody responses to mitogens and antigens [33, 34], prolonged homograft survival of skin [35], enhanced development of tumors [36] and increased susceptibility to infections [37, 38]. These immunological changes have been attributed to pregnancy-associated factors such as early pregnancy factor, placental protein 14, AFP, hormonal steroids, estrogens and progesterones, pre-implantation factor and pregnancy-specific glycoproteins [39-42]. AFP is associated with the normal growth of the mammalian fetus. During fetal life, AFP is produced mainly by the embryonic yolk sac and liver. This serum glycoprotein, produced in high concentrations during embryonic and neonatal stages, tends to decline drastically as the organism matures.

We suggested that some of these observed immunomodulating effects on autoimmune diseases might be related to the high levels in the maternal serum of AFP [43]. Comprehensive research done in our laboratory, using human AFP isolated from amniotic fluid $[33,34,43]$, and recombinant human AFP isolated from Escherichia coli or from the milk of transgenic goats [44], revealed broad immunomodulatory activity, influencing autoimmune disorders and inflammatory reactions. We have studied AFP in two major animals models, EAE and experimental autoimmune myasthenia gravis (EAMG). The latter serves as a model for MG and is an antibody-mediated autoimmune disease caused by specific antibodies to $\mathrm{AChR}$ at the neuromuscular junction.

Following AFP treatment (3-10 $\mu \mathrm{g} / \mathrm{mouse} /$ day, i.p.) a dramatic amelioration of all the clinical parameters of

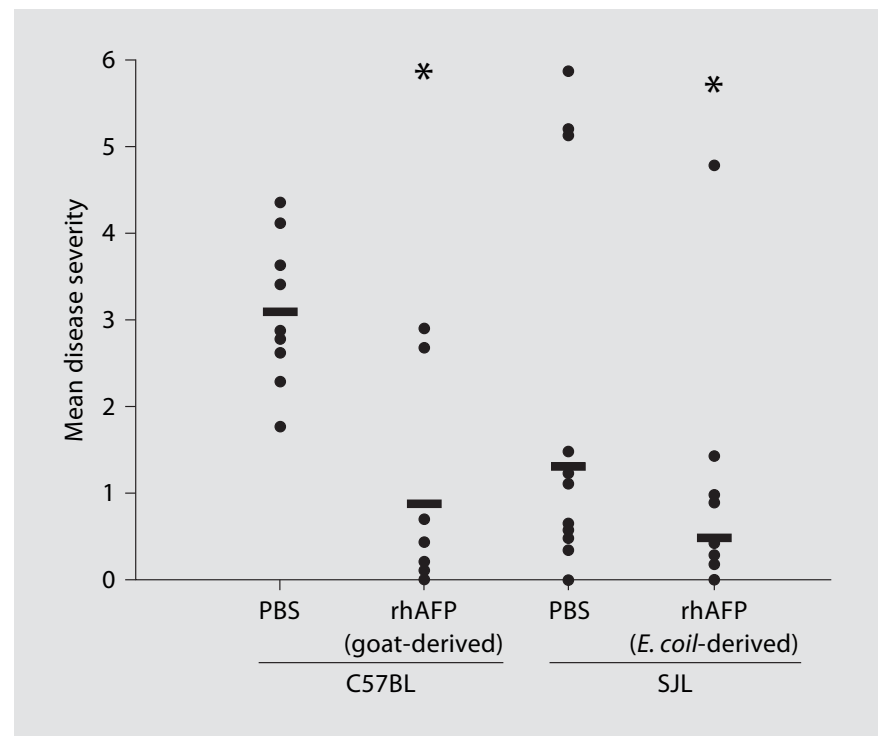

Fig. 3. Treatment with rhAFP suppresses EAE. C57BL/6 female mice immunized with MOG 35-55/CFA were treated with goatderived rhAFP $(10 \mu \mathrm{g} / \mathrm{mouse} /$ day, i.p.) from the day of disease induction. SJL female mice immunized with mouse spinal cord homogenate/CFA were treated with E. coli-derived rhAFP $(3 \mu \mathrm{g} /$ mouse/day, i.p.). The results are of a representative experiment and are expressed as the mean clinical score for each mouse. ${ }^{*} \mathrm{p}<0.05$.

EAE was observed. The mean severity was reduced by $65-70 \%$, as can be seen in figure 3. This prominent effect was obtained in both C57BL and SJL mouse species. It appears that the suppressive in vivo effect of AFP was biphasic: complete protection in animals that did not show clinical symptoms, and partial protection of those that developed a milder disease. The clinical findings were consistent with the histological manifestation of CNS sections. Bielschowsky silver impregnation combined with hematoxylin staining revealed marked dystrophy and loss of axons, together with massive infiltration of mononuclear cells in EAE mice treated with PBS (fig. 4). Furthermore, treatment with recombinant human (rhAFP) markedly limited the inflammatory process and axonal pathology (fig. 4). rhAFP reduced the extent of axonal injury by $55 \%$, from a mean value of 1.6 \pm 0.12 in the PBS-treated mice to $0.9 \pm 0.09$ in the rhAFP-treated mice (scale: $0=$ normal, $1=$ scattered injured axons, $2=$ mild to moderate axonal injury, $3=$ severe axonal injury). Moreover, rhAFP limited the axonal loss by up to $85 \%$, from a mean value of $0.5 \pm 0.06$ in the PBS-treated mice to $0.1 \pm 0.06$ in the rhAFP-treated mice (scale: $0=$ normal, $1=$ mild to moderate axonal 

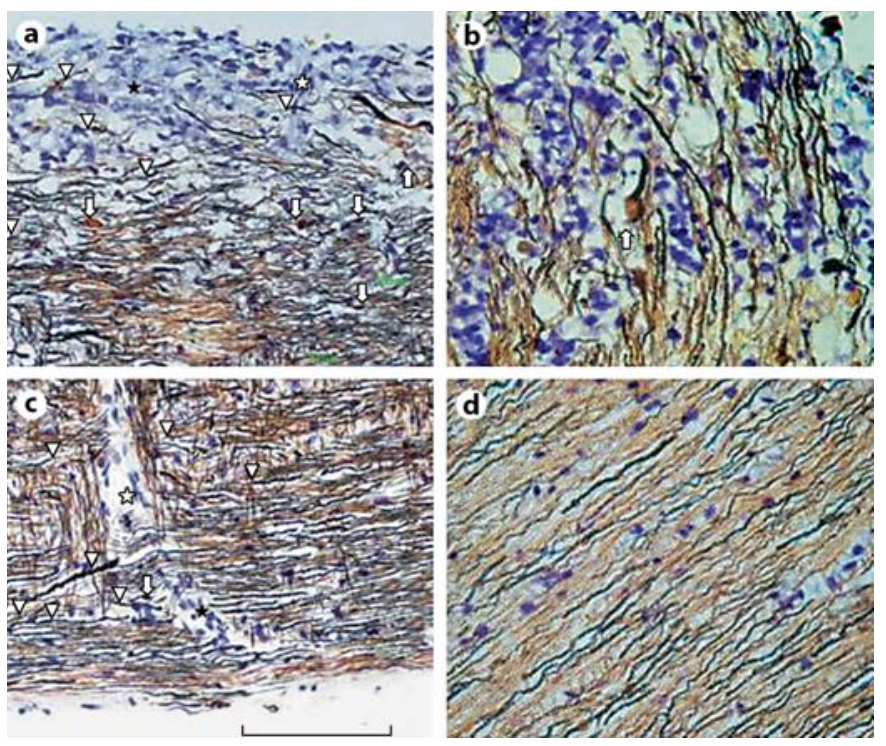

Fig. 4. AFP reduces axonal damage and inflammatory infiltrates in spinal cord tissue from EAE mice. a, b Bielschowsky silver impregnation combined with hematoxylin staining revealed marked dystrophy and loss of axons (low density of brown-stained axons), together with massive infiltration of mononuclear cells in EAE mice treated with PBS. c, d Following treatment with rhAFP (10 $\mu \mathrm{g} / \mathrm{mouse} /$ day, i.p.) both axonal pathology and inflammation were limited. In all figures: open asterisks indicate the vessel; dark asterisks indicate the areas of inflammation; arrows indicate the spheroids or ovoids (features of injured axons); arrowheads indicate dilated axons (another feature of degenerated injured axons). Magnification: a, $\mathbf{c} \times 40$; b, $\mathbf{d} \times 100$.

loss, 2 = severe axonal loss). A comparison of the number of inflammatory infiltrates showed $76 \%$ reduction following rhAFP treatment $(2.3 \pm 1.3$ compared with $9.8 \pm 1.2$ in the PBS-treated mice). The minimal inflammation observed in the AFP-treated animals was accompanied by an increased number of mononuclear apoptotic cells, as revealed by caspase- 3 and fractin immunostaining.

$T$ cells play a central role in the pathogenesis of EAE, both at the initial phase in the periphery and later as the inflammatory process becomes limited to the CNS. Influencing this arm of the immune response is of prime importance in the treatment of autoimmune disorders. In our study, treatment with AFP suppressed T-cell reactivity towards the MOG encephalitogenic peptide 35-55, as manifested by a significant reduction in the proliferation rate associated with lower secretion of IFN- $\gamma$ and TNF- $\alpha$, both proinflammatory cytokines extensively involved in EAE and MS (fig. 5). This ability of AFP may be

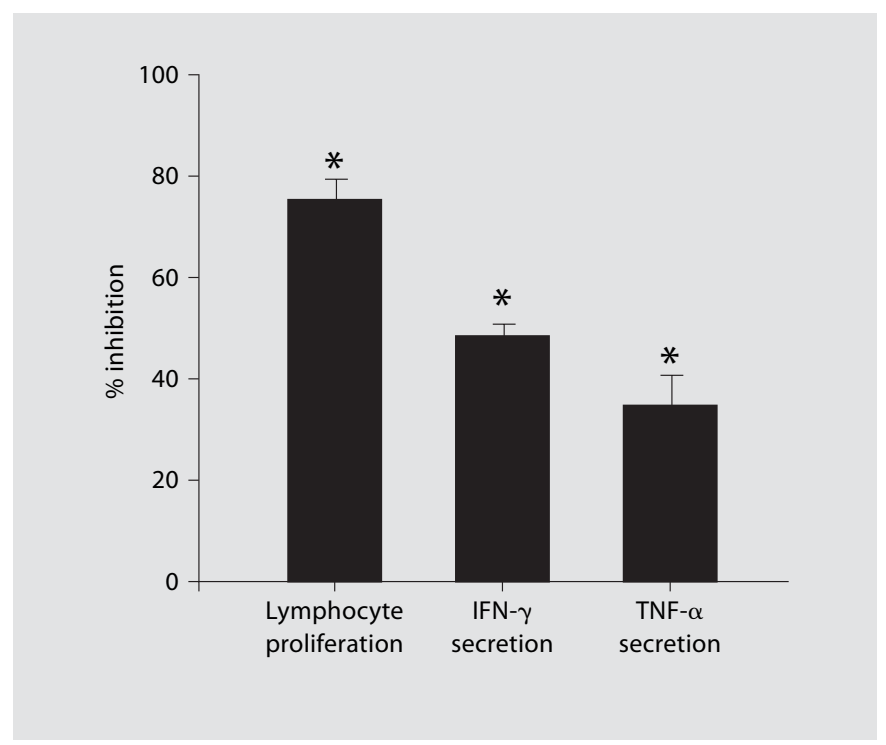

Fig. 5. Inhibitory effect of rhAFP on T-cell reactivity ex vivo. Lymphocytes from PBS and rhAFP-treated (10 $\mu \mathrm{g} /$ day for 9 days) MOG-induced EAE mice were analyzed for proliferation, IFN- $\gamma$ and TNF- $\alpha$ secretion (after $4 \mathrm{~h}$ in culture) by MOG encephalitogenic peptide $35-55(100 \mu \mathrm{g} / \mathrm{ml})$. The data are expressed as percent inhibition relative to the PBS-treated group. ${ }^{*} \mathrm{p}<0.05$.

related to the disrupted antigen presentation, manifested by downregulation of MHC class II expression, observed following treatment. The effect of AFP was restricted to activated lymphocytes, supporting previous studies that identified specific receptors for AFP on activated lymphocytes but not on resting cells $[45,46]$. Moreover, following treatment with AFP there was a decline of $57 \%$ in the number of lymphocytes expressing the chemokine receptor CCR5, which mediates extravasation of inflammatory cells from the circulation into the CNS through the blood vessel endothelial wall. Thus, by reducing their proliferation and altering CCR5 expression, AFP can prevent the organ-specific migration of encephalitogenic T cells to the CNS, as well as ameliorate local inflammation. The beneficial effect of AFP on CNS inflammation and demyelination may also be attributed to its ability to suppress the production of myelin-specific antibodies which are also involved in the immune cascade leading to axonal damage. Indeed, a 60 and $70 \%$ reduction in MOG-specific IgM and IgG antibodies, respectively, was observed following rhAFP treatment. The ability of AFP to regulate immune reactions was not restricted to EAE. Treatment with AFP of EAMG Lewis rats improved the clinical outcome of the animals. 
We conclude that the embryo-specific glycoprotein AFP induces a spectrum of immunomodulating effects, influencing several components of the immune system that participate in the pathology of EAE. As mentioned above, AFP caused reduced T- and B-lymphocyte reactivity together with altered antigen presentation and cell $\mathrm{mi}$ gration. The ability of a natural molecule such as AFP to exert such multi-level non-toxic activity, points to its potential use for the treatment of MS and other autoimmune and inflammatory disorders.

To summarize, we describe two novel strategies for coping with neuroinflammation: (1) Bifunctional com- pounds that enable multiple targeting and enhanced CNS permeability. The unique pharmacodynamic profile of these molecules renders them superior to combination therapy. (2) The use of AFP, a pregnancy-associated protein that exerts a multi-level effect on the various stages of EAE pathogenesis. Both strategies can be, and in the case of naturally occurring compounds are, utilized in many more clinical settings. Our data suggest that drug development productivity may be increased not only by 'reinventing the wheel', but also by a more judicious use of that which already exists.

\section{References}

1 Schmid EF, Smith DA: Keynote review: Is declining innovation in the pharmaceutical industry a myth? Drug Discov Today 2005;10: 1031-1039.

-2 Van der Greef J, McBurney RN: Innovation: Rescuing drug discovery: in vivo systems pathology and systems pharmacology. Nat Rev Drug Discov 2005;4:961-967.

-3 Kramer R, Cohen D: Functional genomics to new drug targets. Nat Rev Drug Discov 2004; 3:965-972.

4 Torr-Brown S: Advances in knowledge management for pharmaceutical research and development. Curr Opin Drug Discov Devel 2005;8:316-322.

5 Steinman L, Zamvil SS: How to successfully apply animal studies in experimental allergic encephalomyelitis to research on multiple sclerosis. Ann Neurol 2006;60:12-21.

-6 Hemmer B, Archelos JJ, Hartung HP: New concepts in the immunopathogenesis of multiple sclerosis. Nat Rev Neurosci 2002;3: 291-301.

7 Steinman L: Multiple sclerosis: a two-stage disease. Nat Immunol 2001;2:762-764.

-8 Bjartmar C, Wujek JR, Trapp BD: Axonal loss in the pathology of MS: consequences for understanding the progressive phase of the disease. J Neurol Sci 2003;206:165-171.

9 Amitai G, Adani R, Rabinovitz I, Beit-Yanai E, Shohami E, Sod-Moriah G, Meshulam H: Cholinergic Mechanisms: Function and Dysfunction, Chapt 41. Newgen Imaging Systems, 2004.

-10 Farias GG, Godoy JA, Vazquez MC, Adani R, Meshulam H, Avila J, Amitai G, Inestrosa NC: The anti-inflammatory and cholinesterase inhibitor bifunctional compound IBU-PO protects from $\beta$-amyloid neurotoxicity by acting on Wnt signaling components. Neurobiol Dis 2005;18:176-183.

11 Drews J: Case histories, magic bullets and the state of drug discovery. Nat Rev Drug Discov 2006;5:635-640.
2 Kanwar JR, Kanwar RK, Krissansen GW: Simultaneous neuroprotection and blockade of inflammation reverses autoimmune encephalomyelitis. Brain 2004;127:1313-1331.

13 Nizri E, Adani R, Meshulam H, Amitai G, Brenner T: Bifunctional compounds eliciting both anti-inflammatory and cholinergic activity as potential drugs for neuroinflammatory impairments. Neurosci Lett 2005; 376:46-50.

14 Moon C, Ahn M, Wie MB, Kim HM, Koh CS, Hong SC, Kim MD, Tanuma N, Matsumoto Y, Shin T: Phenidone, a dual inhibitor of cyclooxygenases and lipoxygenases, ameliorates rat paralysis in experimental autoimmune encephalomyelitis by suppressing its target enzymes. Brain Res 2005;1035:206210.

15 Weggen S, Eriksen JL, Das P, Sagi SA, Wang R, Pietrzik CU, Findlay KA, Smith TE, Murphy MP, Bulter T, Kang DE, Marquez-Sterling N, Golde TE, Koo EH: A subset of NSAIDs lower amyloidogenic A $\beta 42$ independently of cyclooxygenase activity. Nature 2001;414:212-216.

16 Scheuren N, Bang H, Munster T, Brune K, Pahl A: Modulation of transcription factor NF- $\kappa \mathrm{B}$ by enantiomers of the non-steroidal drug ibuprofen. Br J Pharmacol 1998;123: 645-652.

17 Barnes PJ, Karin M: Nuclear factor-кB: a pivotal transcription factor in chronic inflammatory diseases. N Engl J Med 1997; 336:1066-1071.

18 Sacco S, Agnello D, Sottocorno M, Lozza G, Monopoli A, Villa P, Ghezzi P: Nonsteroidal anti-inflammatory drugs increase tumor necrosis factor production in the periphery but not in the central nervous system in mice and rats. J Neurochem 1998;71:2063-2070.
Wang H, Yu M, Ochani M, Amella CA, Tanovic M, Susarla S, Li JH, Yang H, Ulloa L, Al-Abed Y, Czura CJ, Tracey KJ: Nicotinic acetylcholine receptor $\alpha 7$ subunit is an essential regulator of inflammation. Nature 2003;421:384-388.

20 Nizri E, Hamra-Amitay Y, Sicsic C, Lavon I, Brenner T: Anti-inflammatory properties of cholinergic up-regulation: a new role for acetylcholinesterase inhibitors. Neuropharmacology 2006;50:540-547.

-21 Simpson SH, Eurich DT, Majumdar SR, Padwal RS, Tsuyuki RT, Varney J, Johnson JA: A meta-analysis of the association between adherence to drug therapy and mortality. BMJ 2006;333:15.

22 Miller NH, Hill M, Kottke T, Ockene IS: The multilevel compliance challenge: recommendations for a call to action. A statement for healthcare professionals. Circulation 1997;95:1085-1090.

23 DiMatteo MR: Variations in patients' adherence to medical recommendations: a quantitative review of 50 years of research. Med Care 2004;42:200-209.

24 O'Connor KA, Roth BL: Finding new tricks for old drugs: an efficient route for publicsector drug discovery. Nat Rev Drug Discov 2005;4:1005-1014

25 Salha O, Balen AH: New concepts in superovulation strategies for assisted conception treatments. Curr Opin Obstet Gynecol 2000; 12:201-206.

26 Carrel AL, Allen DB: Effects of growth hormone on body composition and bone metabolism. Endocrine 2000;12:163-172.

27 Offner H: Neuroimmunoprotective effects of estrogen and derivatives in experimental autoimmune encephalomyelitis: therapeutic implications for multiple sclerosis. J Neurosci Res 2004;78:603-624.

28 Burrow G, Ferris T: Medical Complications during Pregnancy, Philadelphia, Saunders, 1975. 
29 Da Silva J, Spector T: The role of pregnancy in the course and etiology of rheumatoid arthritis. Clin Rheumatol 1992;11:189-194.

$\checkmark 30$ Olding LB, Papadogiannakis N, Barbieri B, Murgita RA: Suppressive cellular and molecular activities in maternofetal immune interactions; suppressor cell activity, prostaglandins, and $\alpha$-fetoproteins. Curr Top Microbiol Immunol 1997;222:159-187.

-31 Confavreux C, Hutchinson M, Hours MM, Cortinovis-Tourniaire P, Moreau T: Rate of pregnancy-related relapse in multiple sclerosis. Pregnancy in Multiple Sclerosis Group. N Engl J Med 1998;339:285-291.

>32 Ciafaloni E, Massey JM: Myasthenia gravis and pregnancy. Neurol Clin 2004;22:771782 .

-33 Brenner T, Abramsky O, Lisak RP: Influence of $\alpha$-fetoprotein on the in vitro and in vivo immune response to acetylcholine receptor. Ann NY Acad Sci 1981;377:208-221.

-34 Brenner T, Zielinski A, Argov Z, Abramsky $O$ : Prevention of experimental autoimmune myasthenia gravis in rats by fetal $\alpha$-fetoprotein-rich fractions. Tumour Biol 1984;5: 263-274.
-35 Andresen RH, Monroe CW: Experimental study of the behavior of adult human skin homografts during pregnancy. A preliminary report. Am J Obstet Gynecol 1962;84: 1096-1103.

36 Gleicher N, Cohen CJ, Deppe G, Gusberg SB: Familial malignant melanoma of the female genitalia: a case report and review. Obstet Gynecol Surv 1979;34:1-15.

37 Evron S, Brenner T, Abramsky O: Suppressive effect of pregnancy on the development of experimental allergic encephalomyelitis in rabbits. Am J Reprod Immunol 1984;5: 109-113.

38 Morell V: Zeroing in on how hormones affect the immune system. Science 1995;269:773775 .

39 Bebo BF Jr, Dveksler GS: Evidence that pregnancy specific glycoproteins regulate T-cell function and inflammatory autoimmune disease during pregnancy. Curr Drug Targets Inflamm Allergy 2005;4:231-237.

40 Harness J, Cavanagh A, Morton H, McCombe P: A protective effect of early pregnancy factor on experimental autoimmune encephalomyelitis induced in Lewis rats by inoculation with myelin basic protein. J Neurol Sci 2003;216:33-41.

-41 Langer-Gould A, Garren H, Slansky A, Ruiz PJ, Steinman L: Late pregnancy suppresses relapses in experimental autoimmune encephalomyelitis: evidence for a suppressive pregnancy-related serum factor. J Immunol 2002;169:1084-1091.
42 Mishan-Eisenberg G, Borovsky Z, Weber MC, Gazit R, Tykocinski ML, Rachmilewitz J: Differential regulation of Th1/Th2 cytokine responses by placental protein 14 . J Immunol 2004;173:5524-5530.

43 Abramsky O, Brenner T, Mizrachi R, Soffer D: Alpha-fetoprotein suppresses experimental allergic encephalomyelitis. J Neuroimmunol 1982;2:1-7.

44 Irony-Tur-Sinai M, Grigoriadis N, Lourbopoulos A, Pinto-Maaravi F, Abramsky O, Brenner T: Amelioration of autoimmune neuroinflammation by recombinant human $\alpha$-fetoprotein. Exp Neurol 2006;198:136144.

45 Suzuki Y, Zeng CQ, Alpert E: Isolation and partial characterization of a specific $\alpha$-fetoprotein receptor on human monocytes. J Clin Invest 1992;90:1530-1536.

46 Torres JM, Laborda J, Naval J, Darracq N, Calvo M, Mishal Z, Uriel J: Expression of $\alpha$ fetoprotein receptors by human T lymphocytes during blastic transformation. Mol Immunol 1989;26:851-857. 